\title{
GPPS-CH-2020-0098
}

\section{PREDICTION OF TURBULENT AXISYMMETRIC FLOWS USING AN ARTIFICIAL COMPRESSIBILITY APPROACH}

\author{
Georgios N. Lygidakis \\ Technical University of Crete, School of \\ Production Engineering and Management \\ glygidakis@isc.tuc.gr \\ Chania, Crete, Greece \\ Anargiros I. Delis \\ Technical University of Crete, School of \\ Production Engineering and Management \\ adelis@science.tuc.gr \\ Chania, Crete, Greece
}

\author{
Stavros N. Leloudas \\ Technical University of Crete, School of \\ Production Engineering and Management \\ sleloudas@isc.tuc.gr \\ Chania, Crete, Greece \\ loannis K. Nikolos \\ Technical University of Crete, School of \\ Production Engineering and Management \\ jnikolo@dpem.tuc.gr \\ Chania, Crete, Greece
}

\begin{abstract}
Swirling flows are revealed to be of crucial importance across a wide range of engineering applications. Especially in the field of turbomachines; the ability to efficiently predict the characteristics of such flows has a significant impact on their design. Motivated by the previous state, the in-house academic solver IGal2D is presented in this study, that has been recently enhanced to simulate swirling incompressible flows. Flow modeling is based on the modified, by the artificial compressibility approach, axisymmetric ReynoldsAveraged Navier-Stokes equations (including tangential velocity) and Shear Stress Transport turbulence model, expressed in cylindrical coordinates. Discretization is achieved via two-dimensional hybrid unstructured grids, composed of triangular and quadrilateral elements, along with a node-centered finite-volume scheme. For the computation of inviscid fluxes, the Roe approximate Riemann solver is implemented, coupled with a higher-order accurate spatial scheme, whereas for viscous ones the required gradients are evaluated with an element-based approach. Time integration is succeeded through the secondorder temporal accurate four-stage Runge-Kutta method. For the improvement of its computational performance an agglomeration multigrid scheme is utilized along with an edge-based data structure and a local time-stepping technique. The proposed solver is validated against relevant challenging test cases considering inviscid and viscous, laminar and turbulent, flows. The extracted results are compared with those of the commercial software ANSYS Fluent, confirming the proposed methodology's potential to predict such flows in terms of accuracy.
\end{abstract}

\section{INTRODUCTION}

During the last decades, the escalating awareness for climate change has led to a revision of the conventional energy model and simultaneously to a wider adoption of sustainable energy technologies based on renewable resources, e.g. wind turbines. Besides large scale wind farm installations, small-scale wind energy conversion systems have attracted attention from the international engineering community since they represent a promising solution for sustainable energy production in site-specific cases. In particular, a significant effort has been exerted for the development of diffuser-augmented or shrouded wind turbines, due to the flexibility they provide both in terms of required space and wind speed conditions, i.e. they can be integrated within a much broader spectrum of residential, rural and remote areas (Leloudas et al., 2020a; Leloudas et al., 2020b; Singh et al., 2012; Yang et al., 2019).

Computational Fluid Dynamics (CFD) has been revealed as an indispensable tool to the development process of the aforementioned small-scale wind energy conversion systems, mainly due to its capability to predict aerodynamic behavior of wind turbine configurations against different wind conditions in a relatively short period of time. Therefore, the designers rely strongly on the results of the corresponding algorithms, as in that way they may avoid, initially at least, a large fraction of the extremely time and money consuming experiments. Considering the effectiveness of CFD simulations, quite many numerical solvers have been developed in the past years for the prediction of incompressible fluid flow, whose differences concern various 
issues, e.g., grid type, discretization method, flux evaluation, turbulence modeling, etc. (Blazek, 2001; Lygidakis et al., 2016).

Although three-dimensional CFD calculations have become relatively commonplace nowadays, mainly due to the advance of the available computing systems, twodimensional axisymmetric flow solvers are still considered as highly valuable tools, especially in the field of turbomachinery (including wind turbines). In such codes, curvilinear grids are applied in the axial and radial directions, whereas no grid is used in the azimuthal one. Each control volume is represented as a body of revolution with constant two-dimensional cross-sectional areas in the azimuthal direction. Thus, axisymmetric algorithms allow for a threedimensional flow problem to be approximated with a twodimensional one and consequently for significant reduction in the required computational time and pre-processing effort (Leloudas et al., 2020a; Sheng-Tao, 1993).

Swirling or rotating flows, commonly occur in both nature and engineering applications, is part of the application field of the aforementioned CFD solvers. Over the past years, engineers have strived to reproduce and control swirls where they are favorable to their applications and suppress, with great difficulty, the occurrence of swirl flows where they are undesirable. Consequently, great efforts have been exerted for the development of axisymmetric codes, including additionally a transport equation for the tangential velocity, in order the circumferentially averaged flow field in the case of a three-dimensional swirling flow to be reliably predicted along with non-trivial savings in both computing time and resources (Resiga et al., 2009; Rocha et al., 2015).

Motivated by the previous states, the enhancement of the in-house academic CFD solver IGal2D (of the Galateafamily software) to simulate axisymmetric swirling incompressible flows is reported in this study (Leloudas et al., 2020a; Leloudas et al., 2020b; Lygidakis et al., 2016). An additional transport equation for the circumferential velocity is taken into account with analogous modifications in axisymmetric Reynolds-Averaged Navier-Stokes (RANS) equations and Shear Stress Transport (SST) turbulence model, expressed in cylindrical coordinates and dimensionless formulation. Attention is mainly directed towards the accurate prediction of velocity profiles in cases of internal swirling incompressible flows, such as those meet in shrouded wind turbines. To this end, the proposed code is validated against relevant test cases considering inviscid, viscous laminar and viscous turbulent, axially induced swirling pipe flows. The obtained results are compared with those of the commercial CFD software ANSYS Fluent, confirming the proposed methodology's potential to predict such flows in terms of accuracy.

The proposed solver is based on well-established methodologies, namely artificial compressibility approach, unstructured mesh implementation and axisymmetric formulation. Although axisymmetric flow models have lately become a highly attractive topic in the context of mesoscopic approaches, to the authors' best knowledge, the development of an axisymmetric Navier-Stokes solver for swirling flows using the artificial compressibility method is not available in the literature.

The structure of this paper is as follows: Next sections include a brief description of the flow solver, focusing on the governing equations' formulation, spatial and temporal discretization schemes, flux computation, numerical solution, and the main acceleration technique. The rest of the study contains the aforementioned test cases for axially induced swirling pipe flows along with the respective results, which are compared with those of the Fluent software. The corresponding conclusions are analyzed in the final Section.

\section{FLOW MODELING}

\section{Governing Equations}

Flow modeling is based on the modified, by the artificial compressibility approach, axisymmetric RANS PDEs (Partial Differential Equations), described in dimensionless formulation as follows (Leloudas et al., 2020a; Lygidakis et al., 2016)

$$
\frac{\partial \overrightarrow{\mathrm{U}}}{\partial t}+\frac{\partial \overrightarrow{\mathrm{F}}_{i n v}}{\partial x}+\frac{\partial \overrightarrow{\mathrm{G}}_{i n v}}{\partial y}-\frac{\partial \overrightarrow{\mathrm{F}}_{v i s}}{\partial x}-\frac{\partial \overrightarrow{\mathrm{G}}_{v i s}}{\partial y}=\overrightarrow{\mathrm{H}} .
$$

The vector of primitive variables, i.e. pressure and velocity components (axial, radial and tangential), is defined as (Resiga et al., 2009)

$$
\vec{U}=\left[\begin{array}{llll}
p & u & v & w
\end{array}\right]^{T},
$$

whereas those of inviscid and viscous fluxes as

$$
\overrightarrow{\mathrm{F}}_{i n v}=\left[\begin{array}{c}
\beta u \\
u^{2}+p \\
u v \\
u w
\end{array}\right], \quad \overrightarrow{\mathrm{G}}_{i n v}=\left[\begin{array}{c}
\beta v \\
v u \\
v^{2}+p \\
v w
\end{array}\right] .
$$

and

$$
\overrightarrow{\mathrm{F}}_{v i s}=\left[\begin{array}{c}
0 \\
\tau_{x x} \\
\tau_{x y} \\
\tau_{x \theta}
\end{array}\right], \quad \overrightarrow{\mathrm{G}}_{v i s}=\left[\begin{array}{c}
0 \\
\tau_{x y} \\
\tau_{y y} \\
\tau_{y \theta}
\end{array}\right] .(4)
$$

In addition, $\beta$ denotes the artificial compressibility parameter, enhancing the governing equations with hyperbolic characteristics, whereas $\tau$ the viscous shear stresses, computed as follows (Lee and Lee, 2011; Leloudas et al., 2020a; Lygidakis et al., 2016):

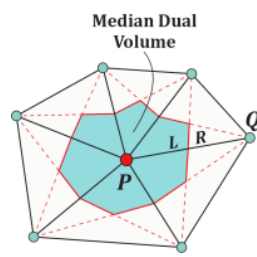

(a)

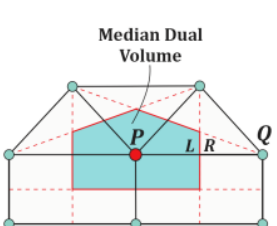

(b) (c)

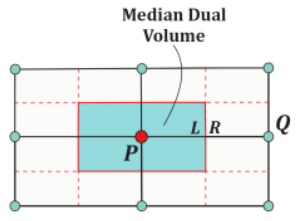

Figure 1 Control Volume of a Node $P$ 


$$
\begin{aligned}
\tau_{x x} & =2\left(\frac{v_{l}}{\operatorname{Re}}+v_{t}\right) \frac{\partial u}{\partial x}-\frac{2}{3} k \\
\tau_{y y} & =2\left(\frac{v_{l}}{\operatorname{Re}}+v_{t}\right) \frac{\partial v}{\partial y}-\frac{2}{3} k \\
\tau_{x y} & =\left(\frac{v_{l}}{\operatorname{Re}}+v_{t}\right)\left(\frac{\partial u}{\partial y}+\frac{\partial v}{\partial x}\right) \\
\tau_{\theta \theta} & =2\left(\frac{v_{l}}{\operatorname{Re}}+v_{t}\right) \frac{v}{y}-\frac{2}{3} k \\
\tau_{x \theta} & =\left(\frac{v_{l}}{\operatorname{Re}}+v_{t}\right) \frac{\partial w}{\partial x} \\
\tau_{y \theta} & =\left(\frac{v_{l}}{\operatorname{Re}}+v_{t}\right)\left(\frac{\partial w}{\partial y}-\frac{w}{y}\right)
\end{aligned}
$$

The laminar and turbulent viscosities are denoted with $v_{l}$ and $v_{t}$, whereas $R e$ and $k$ denote the Reynolds number and turbulent kinetic energy, respectively. Finally, the vector of the total source term is calculated as follows (ANSYS Fluent, 2009; Lee and Lee, 2011; Resiga et al., 2009):

$$
\vec{H}=\frac{1}{y}\left[\begin{array}{c}
-\beta v \\
\tau_{x y}-u v \\
-v^{2}+\tau_{y y}-\tau_{\theta \theta}+w^{2} \\
2\left(\tau_{y \theta}-v w\right)
\end{array}\right] .
$$

Turbulence modeling and subsequently computation of turbulent viscosity and kinetic energy, via which flow and turbulence equations interact, is succeeded with the SST model; a combination actually of the well-established $k-\varepsilon$ and $k$ - $\omega$ models, disregarding their major shortcomings. For its description a similar differential relation to Eq. (1) is used, where no additional model has been incorporated to simulate transition (Leloudas et al., 2020a). Moreover, a loosely coupled approach is followed, according to which the flow and turbulence equations are updated separately (Koobus et al., 2000).

\section{Spatial Discretization and Flux Computation}

The computational domain, represented by unstructured grids composed of triangular and quadrilateral elements, is discretized employing a node-centered finite-volume scheme; the median-dual finite control-volume around a mesh node is constructed by an assembly of linear segments, where each segment is connected to the midpoint of an edge and to the centroid of its neighbouring triangular or quadrilateral element. Such a control-volume (coloured in green), contributed by (a) only triangular elements, (b) by both triangular and quadrilateral elements and (c) by only quadrilateral elements, is illustrated in Fig. 1.

Integrating Eq. (1) over the control volume of each node $P$, the following algebraic equations are derived

$$
\left(\frac{d \vec{U}}{d t}\right)_{P} A_{P}+\sum_{Q \in K_{N}(P) \cup Q_{\text {out }}} \vec{\Phi}_{P Q}^{i n v}-\sum_{Q \in K_{N}(P)} \vec{\Phi}_{P Q}^{v i s}=\vec{H}_{P} A_{P} .
$$

where $A_{P}$ is the area of the control-volume of node $P$ under examination, whereas $Q$ stands for all its neighbouring nodes, connected with an edge.

For the computation of the inviscid fluxes, a onedimensional Riemann problem is assumed at each edge of the examined control-volume (corresponding to an edge $P Q$ ), whereas for its solution Roe's approximate Riemann solver is employed. The evaluation of the fluxes is performed in a single edge-loop, utilizing the edge-based data structure of the proposed code. A second-order spatial accurate reconstruction scheme, based on the MUSCL (Monotonic Upstream Scheme for Conservative Laws) method, enhances the previous calculation (Blazek, 2001; Leloudas et al., 2020a; Lygidakis et al., 2016).

As far as the viscous fluxes are concerned, the gradients of velocity components have to be evaluated previously at the middle of each edge. For this calculation, an elementbased approach is used. It considers the construction of edgedual volumes, including all the elements sharing each examined edge $P Q$. In Fig. 2 an example of such an edgedual volume for different grid types is depicted, including the adjacent elements of an edge $P Q$.
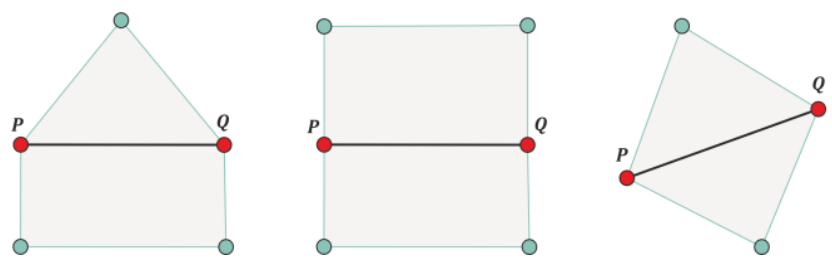

Figure 2 Edge-Dual Volume of an Edge PQ

The necessary gradients are then computed by implementing the divergence theorem on each new control-volume, i.e. on each edge-dual volume (Leloudas et al., 2020a).

Ultimately, appropriate boundary conditions are imposed supplementing the flux balance of the corresponding nodes of the computational mesh or defining straightforward the values of their primitive variables. At each inlet/outlet control-volume, inviscid fluxes are added to its flux balance, derived by the respective normal to the boundary edge vector and vector of Eq. (3). The latter is computed with values of primitive variables taken either from inside or outside the computational domain. In particular, velocity components are taken from outside for inflow and from inside the domain for outflow, whereas for pressure value an opposite strategy is followed (Anderson et al., 1996). Additionally, for the calculation of the pressure distribution over the outlet boundaries, a radial equilibrium assumption is applied. In case of inviscid flows, free-slip conditions are implemented at solid wall regions; the extracted fluxes are added to those of the corresponding boundary nodes. At the same areas, in case of viscous flows, the velocity components are zeroized explicitly (Lygidakis et al., 2016).

The incorporated SST turbulence model is treated similarly. Nevertheless, for the convective fluxes a simple 
upwind scheme is employed with first-order accuracy; in that way stability of the numerical methodology is assured without reducing noticeably the accuracy of the final solution. The diffusive fluxes as well as the necessary gradients are computed in the same way as those of the flow equations, i.e. with the aforementioned element-based approach. In case of a swirling flow, attention should be paid to the tensor $S$, to include the additional terms related to the circumferential velocity, computed as follows (ANSYS Fluent, 2009; Blazek, 2001):

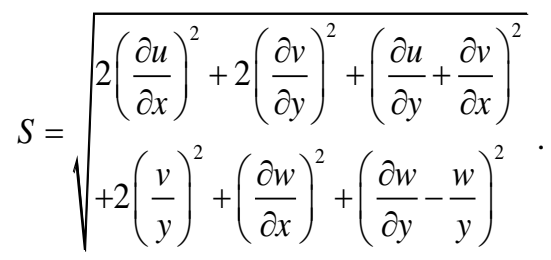

Subsequently, the turbulent energy production term $P_{k}$ is significantly affected as:

$$
P_{k}=v_{t} S^{2}
$$

As far as the corresponding boundary conditions are concerned, they are implemented explicitly at inlet and solid wall surfaces (Dirichlet conditions). In particular, at inflow, values of turbulent variables at far-field are given to the corresponding nodes, whereas at solid walls, turbulent kinetic energy and viscosity are zeroized. On the contrary, at outlet boundaries a simple upwind scheme is imposed, for which data are taken from inside the computational domain.

\section{Numerical Procedure}

After the models' flow and turbulence flux balances have been computed for each control-volume, Eq. (7) is reformulated as

$$
-A_{P} \frac{\Delta \vec{U}_{P}^{k+1}}{\Delta t_{P}}=\vec{R}_{P}^{k} \cdot(10)
$$

where $\Delta U$ denotes the corrections of the variables for the internal time step $k+1$ and $R$ is the calculated summed flux balance at node $P$ at the previous pseudo-time step $k . \Delta t$ represents the pseudo-time step, evaluated with a local timestepping technique to improve the computational performance of the proposed solver (Blazek, 2001). For the calculation of the aforementioned corrections an explicit second-order accurate four-stage Runge-Kutta method (RK(4)) is employed. Finally, as mentioned in the previous section, in case of a turbulent flow a loose-coupling strategy between flow and turbulence models is followed (Lygidakis et al., 2016).

\section{Agglomeration Multigrid Scheme}

Besides the aforementioned acceleration techniques, i.e. the edge-based data structure and the local time-stepping approach, the proposed solver is enhanced with an agglomeration multigrid scheme to reduce the required computation time, especially in turbulent flow simulations. According to this methodology, the solution of the flow problem is relaxed on successively coarser grids, as to achieve an efficient damping of the low-frequency errors. The necessary, progressively coarser, resolutions are obtained via the (isotropic or directional) fusion of the adjacent control-volumes of the finer mesh in a way resembling the advancing front technique. The incorporated full-coarsening directional approach is implemented in quadrilateral regions of hybrid grids. The associating relations between each two successive multigrid levels are obtained with the Full Approximation Scheme (FAS) or the combined Full Multigrid-Full Approximation Scheme (FMG-FAS) approach in a $\mathrm{V}$-cycle process. Turbulence models' equations are treated in the same way to the flow ones.

\section{NUMERICAL RESULTS}

As mentioned in the Introduction, IGal2D code is validated against relevant challenging test cases considering inviscid, viscous laminar and viscous turbulent, axially induced swirling pipe flows. The obtained results are compared qualitatively and quantitatively with those of the commercial CFD software ANSYS Fluent.

\section{Inviscid Swirling Flow}

The first test case considers inviscid incompressible flow inside an S-shaped tube, $2.0 \mathrm{~m}$ long and $0.12 \mathrm{~m}$ size (i.d.). The aforementioned geometry as well as the major dimensions of the tube are illustrated in Fig. 3. Its design was based on a cubic ( $3^{\text {rd }}$ order) B-Spline curve over an open uniform knot vector. The control points of the B-Spline curve representing the centerline of the $\mathrm{S}$-shaped tube are included in Table 1. After the definition of the centerline, the internal and external walls of the flow apparatus were constructed by offsetting the centerline in both directions by $0.06 \mathrm{~m}$. It is noted that no re-dimensionalization was performed for this test case due to the dimensionless formulation of IGal2D.

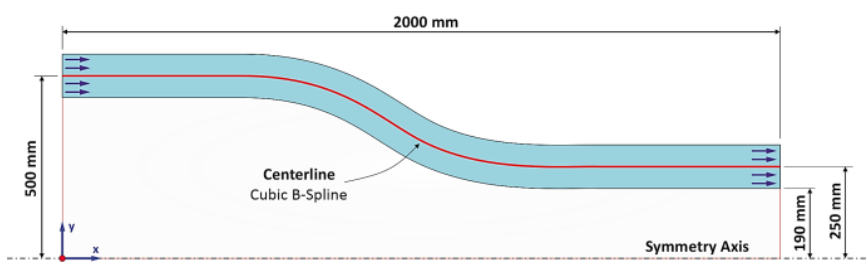

Figure 3 Geometry of the S-shaped Tube

\begin{tabular}{cccccc}
\hline $\boldsymbol{P}_{\boldsymbol{i}}$ & $\boldsymbol{X}_{\boldsymbol{i}}$ & $\boldsymbol{Y}_{\boldsymbol{i}}$ & $\boldsymbol{P}_{\boldsymbol{i}}$ & $\boldsymbol{X}_{\boldsymbol{i}}$ & $\boldsymbol{Y}_{\boldsymbol{i}}$ \\
\hline$P_{0}$ & 0.00000 & 0.50000 & $P_{8}$ & 1.05526 & 0.28803 \\
\hline$P_{1}$ & 0.05259 & 0.50000 & $P_{9}$ & 1.21151 & 0.25478 \\
\hline$P_{2}$ & 0.15775 & 0.50007 & $P_{10}$ & 1.36898 & 0.24795 \\
\hline$P_{3}$ & 0.31554 & 0.49977 & $P_{11}$ & 1.52674 & 0.25055 \\
\hline$P_{4}$ & 0.47310 & 0.50087 & $P_{12}$ & 1.68448 & 0.24985 \\
\hline$P_{5}$ & 0.63114 & 0.49676 & $P_{13}$ & 1.84224 & 0.25004 \\
\hline$P_{6}$ & 0.78490 & 0.45233 & $P_{14}$ & 1.94741 & 0.25000 \\
\hline$P_{7}$ & 0.91876 & 0.36888 & $P_{15}$ & 2.00000 & 0.25000 \\
\hline
\end{tabular}

Table 1 Control Points of the Cubic B-Spline 
For the representation of the computational field, an unstructured mesh of 32,400 nodes and 31,703 quadrilateral elements was constructed. Axial and circumferential velocity at inlet are defined as $0.966 \mathrm{~m} / \mathrm{s}$ and $0.259 \mathrm{~m} / \mathrm{s}$, respectively, whereas relative static pressure at outlet was set equal to 0.0 $\mathrm{Pa}$. The iterative approximation of the final steady-state solution was succeeded with an artificial compressibility parameter equal to 10.0 and a unit CFL number; the pressure residual was decreased more than five orders of magnitude. Finally, two coarser grid resolutions were generated, following the incorporated isotropic agglomeration mode, in order the corresponding multigrid scheme to be exploited. Fig. 4 depicts the obtained contours of dimensional pressure and velocity components, compared with those extracted by ANSYS Fluent where a complete qualitative agreement can be seen between the aforementioned codes.

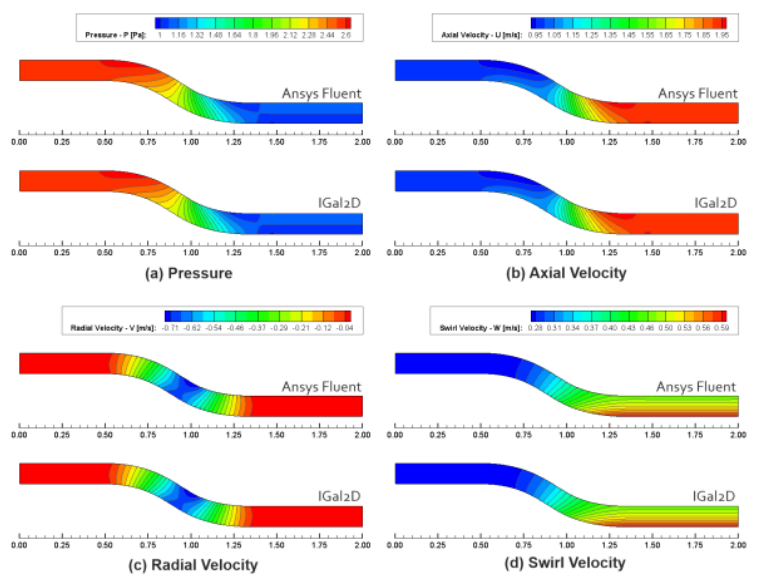

Figure 4 Pressure and Velocity Contours of the Inviscid Flow Inside the S-shaped Tube

Besides the qualitative evaluation of the IGal2D solver, presented above, a quantitative one was performed, comparing the derived dimensional pressure and velocity components distributions at centreline of the examined tube with those of the reference solver. Fig. 5 to 8 illustrate, the actually identical, results confirming the equal potential of the proposed methodology for such internal inviscid incompressible fluid flows in terms of accuracy.

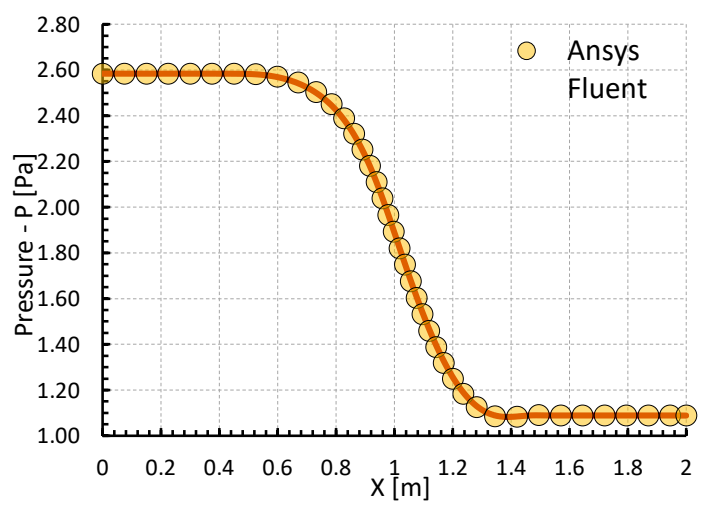

Figure 5 Distribution of Static Pressure $(p)$ Over the Centreline (Inviscid case)

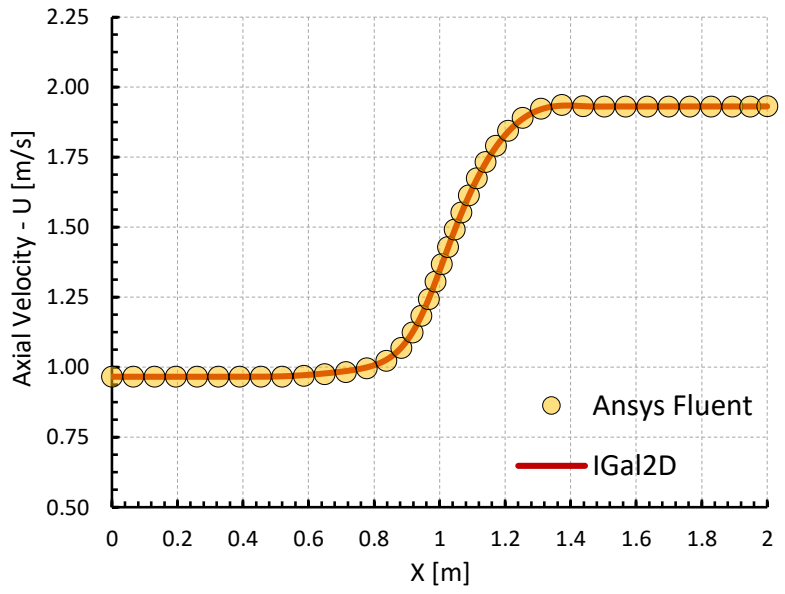

Figure 6 Distribution of the Axial Velocity (u) Over the Centreline (Inviscid case)

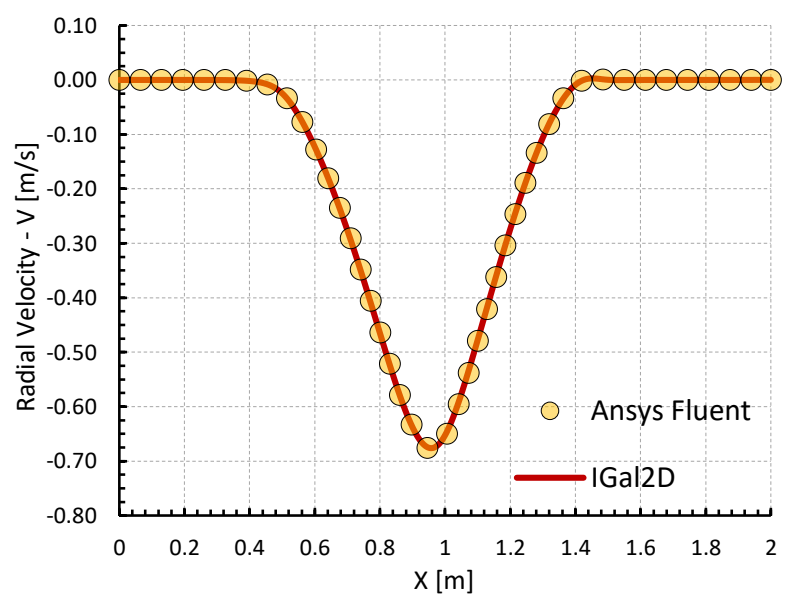

Figure 7 Distribution of the Radial Velocity (v) Over the Centreline (Inviscid case)

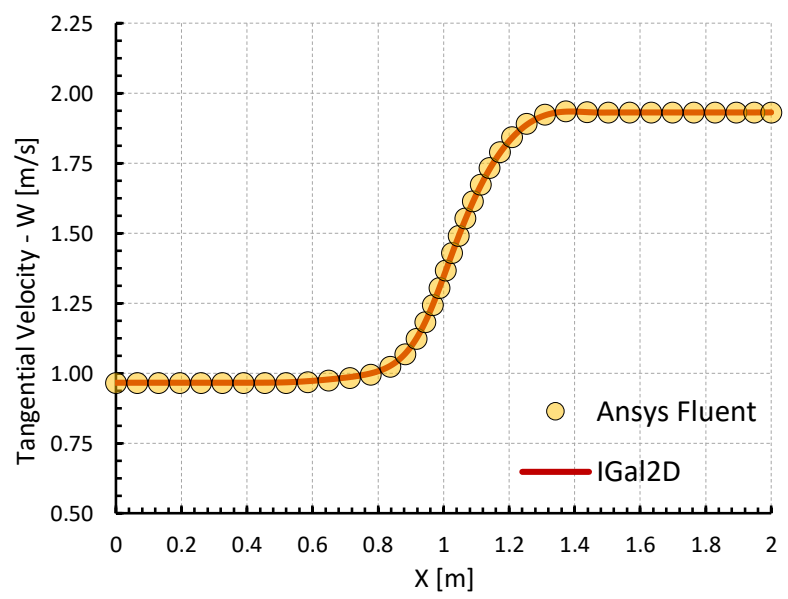

Figure 8 Distribution of the Circumferential (Swirl) Velocity (w) Over the Centreline (Inviscid case)

\section{Laminar Swirling Flow}

The second test case concerns viscous laminar flow in the axial tube following an experimental swirl generator device, reported in (Rocha et al., 2015). The length of the 
pipe is $3.0 \mathrm{~m}$, whereas its diameter is equal to $0.05 \mathrm{~m}$. In addition, it includes a cone (part of the swirl generator) with height $82.0 \mathrm{~mm}$, base diameter $40.0 \mathrm{~mm}$ and deflection angle $63.5^{\circ}$. The whole geometry as well as its dimensions are depicted in Fig. 9. A detailed description can be found in the study of (Rocha et al., 2015). The computational domain is discretized with an unstructured mesh, composed of 59.960 nodes and 58.422 quadrilateral elements and to use it with the proposed dimensionless code, it was re-dimensionalized, such as it's diameter to become equal to unity.

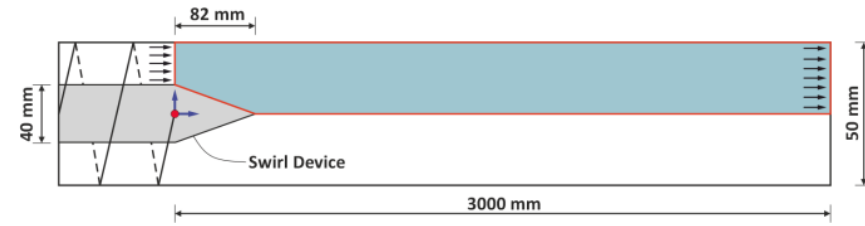

Figure 9 Geometry of the Axial Tube

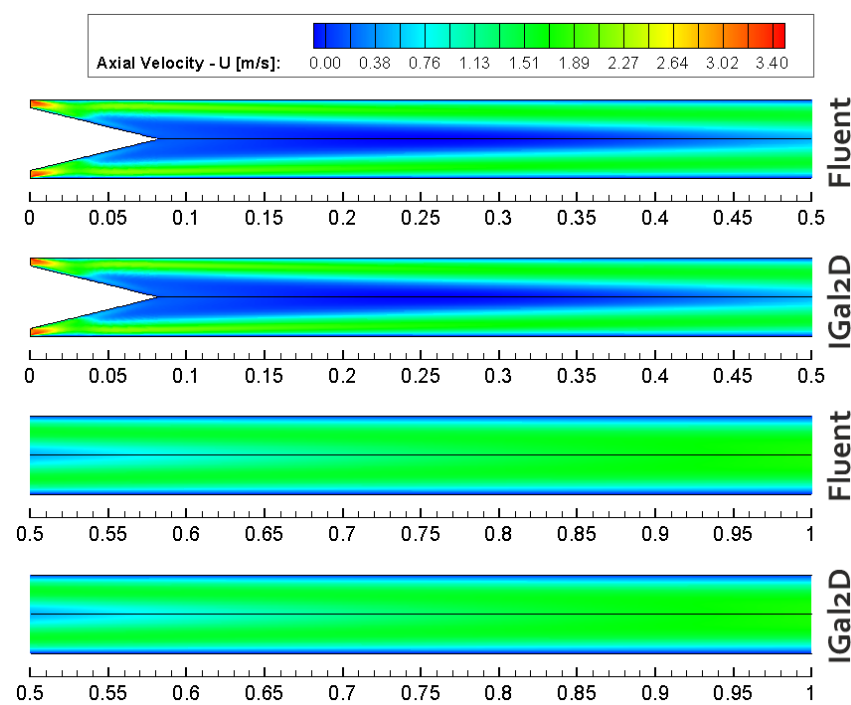

Figure 10 Contours of Axial Velocity

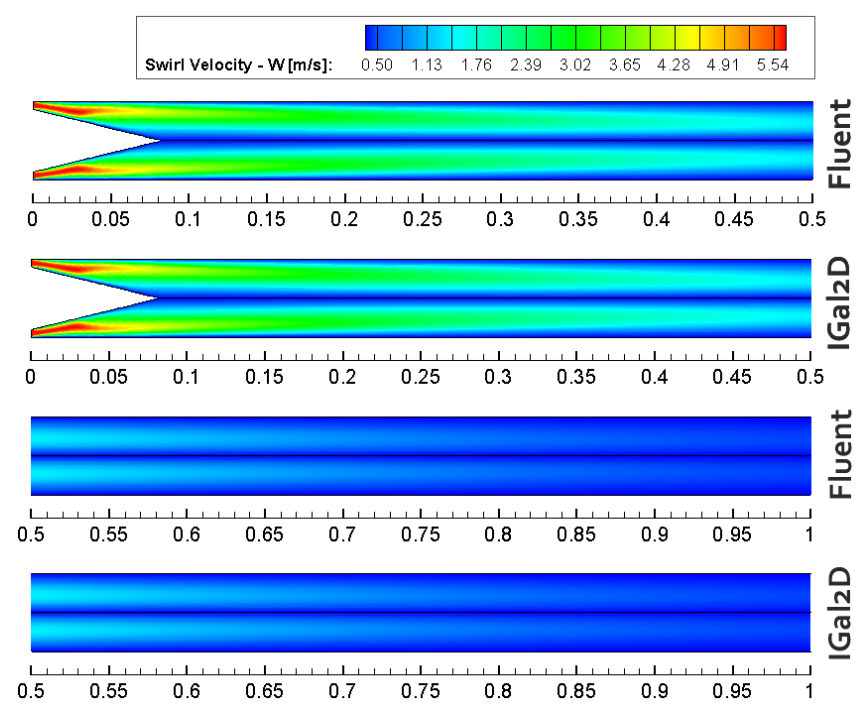

Figure 11 Contours of Swirl Velocity

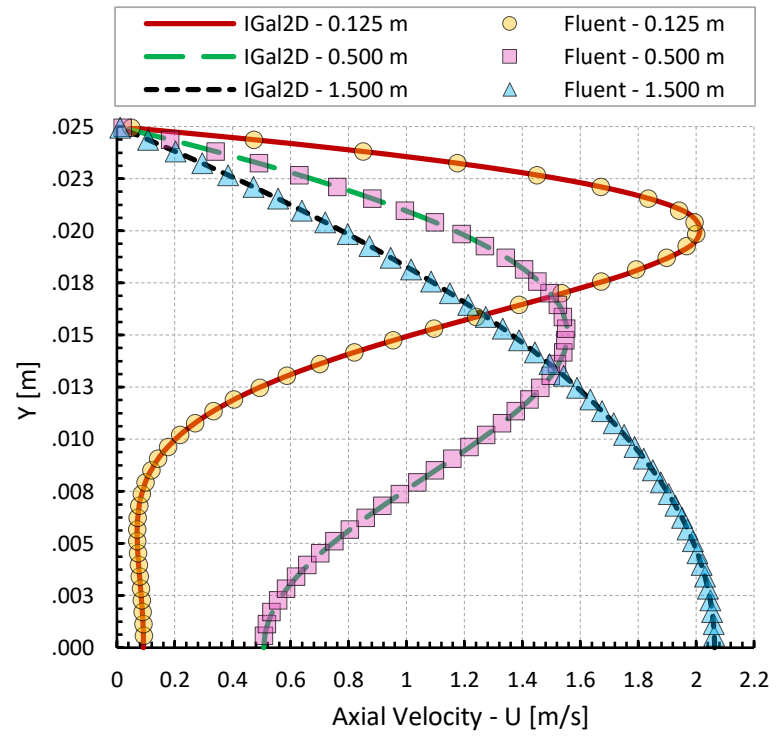

Figure 12 Distributions of Axial Velocity at Different Axial Sections (Laminar case)

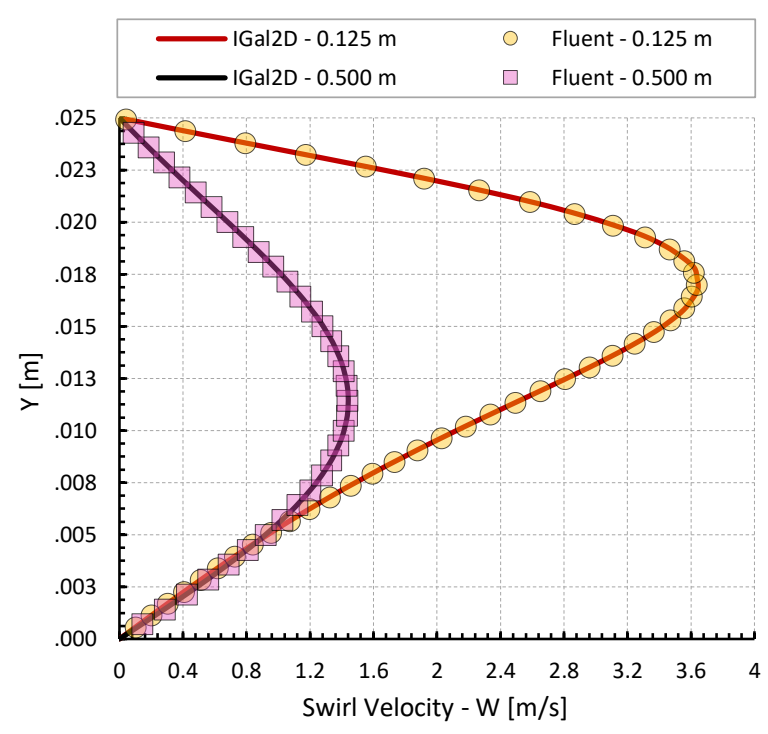

\section{Figure 13 Distributions of Swirl Velocity at Axial} Sections $0.125 \mathrm{~m}$ and $0.5 \mathrm{~m}$ (Laminar case)

Axisymmetric swirling flow is considered, with axial and tangential velocity at inlet $2.947 \mathrm{~m} / \mathrm{s}$ and $5.911 \mathrm{~m} / \mathrm{s}$, respectively. The radial velocity at inlet is $0.0 \mathrm{~m} / \mathrm{s}$, whereas relative static pressure at outlet is equal to $0.0 \mathrm{~Pa}$. As far as the properties of the working fluid are concerned, its density and dynamic viscosity are set equal to $1210 \mathrm{~kg} / \mathrm{m}^{3}$ and 0.216 $\mathrm{kg} / \mathrm{m} / \mathrm{s}$. Based on the aforementioned values the Reynolds number is computed as 1,850 . The final steady-state solution was achieved with a unit artificial compressibility parameter and a CFL number equal to 0.5 ; the pressure residual was decreased more than ten orders of magnitude.

Fig. 10 and 11 illustrate the obtained contours of dimensional axial and swirl velocity, compared with those extracted by ANSYS Fluent; no qualitative difference can be identified between the aforementioned results. Similarly to the previous test case for inviscid flow, a quantitative 
validation was performed with the dimensional axial and circumferential velocity distributions at several different axial sections. Fig. 12 to 14 include these distributions of both compared solvers (IGal2D and Fluent); an almost perfect agreement is achieved, revealing the capability of the proposed code to predict such axisymmetric swirling laminar flows.

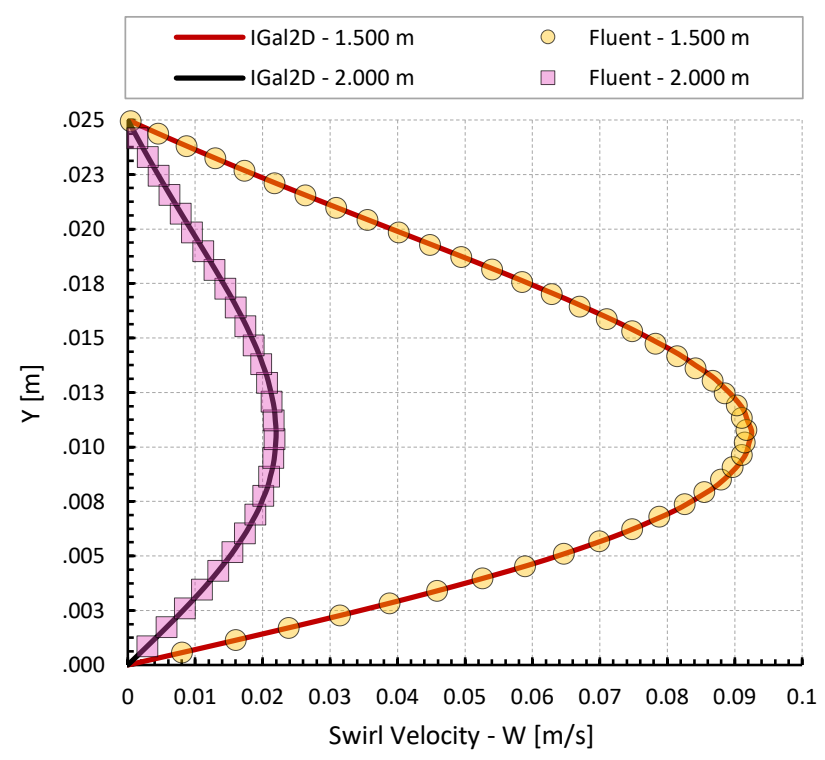

Figure 14 Distribution of Swirl Velocity at Axial Sections $1.5 \mathrm{~m}$ and $2.0 \mathrm{~m}$ (Laminar case)

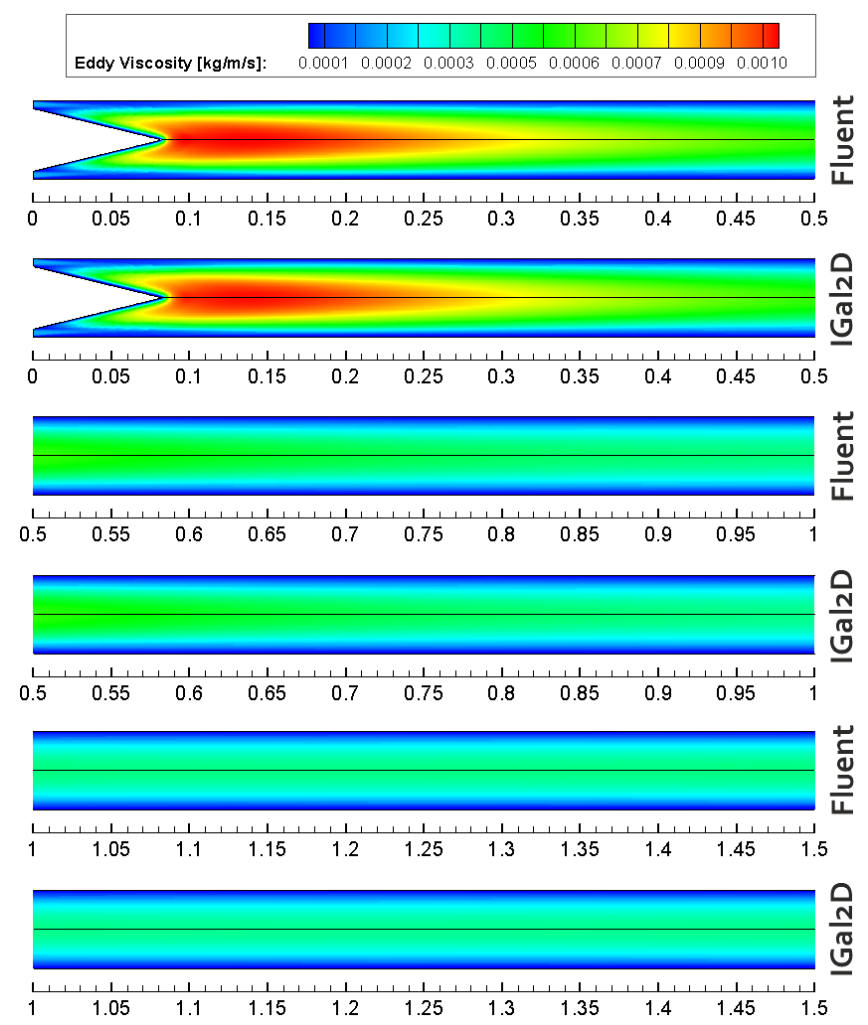

Figure 15 Contours of dynamic eddy viscosity

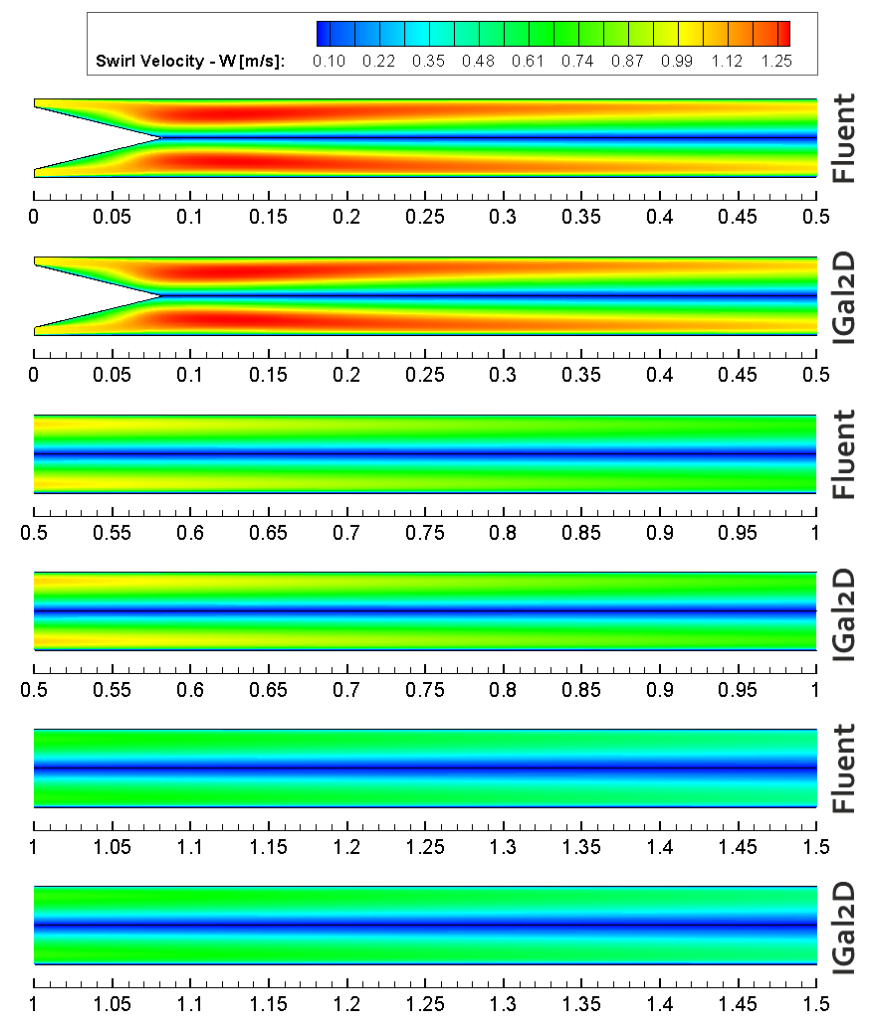

\section{Figure 16 Contours of Swirl Velocity (Turbulent case)}

\section{Turbulent Swirling Flow}

For the last validation case of this study, a viscous turbulent axisymmetric swirling flow in an axial tube, with the geometry of the previous section, is considered. However, a denser grid is used, composed of 109.950 nodes and 107.702 quadrilateral elements and it was redimensionalized similarly, to become suitable for the IGal2D solver. The axial and circumferential velocities at inlet boundary are equal to $5 \mathrm{~m} / \mathrm{s}$ and $1 \mathrm{~m} / \mathrm{s}$, respectively; no radial component is considered again. The relative static pressure at outlet surface is $0.0 \mathrm{~Pa}$. Regarding the properties of the working fluid, its density and dynamic viscosity are set equal to $1.25 \mathrm{~kg} / \mathrm{m}^{3}$ and $0.000018 \mathrm{~kg} / \mathrm{m} / \mathrm{s}$. Based on the aforementioned values the Reynolds number is computed as 17,704.9. The final steady-state solution was succeeded with a unit artificial compressibility parameter and a CFL number equal to 0.5 ; the pressure residual was decreased more than five orders of magnitude.

Fig. 15 and 16 present the derived contours of dimensional kinematic turbulent (eddy) viscosity and swirl velocity, compared with those obtained by ANSYS Fluent. As it can be observed, there is no qualitative difference between the aforementioned results. Subsequent Fig. 17 and 18 include the extracted distributions of dimensional axial and circumferential velocity distributions at several different axial sections. They are almost identical with the corresponding distributions derived by the Fluent software. Thus, the simulation capability of the proposed methodology against such flow phenomena is once more confirmed. 


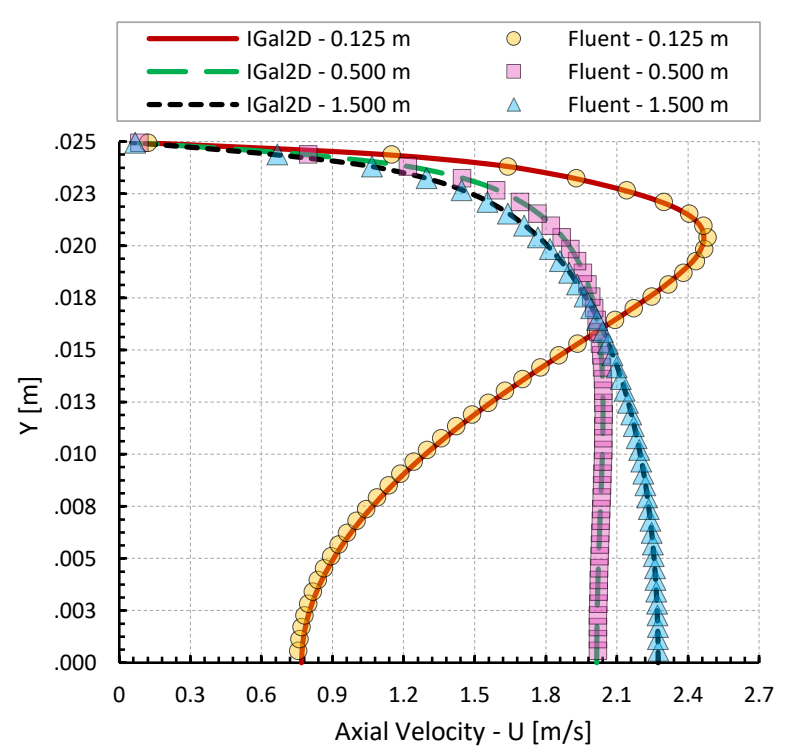

Figure 17 Distributions of Axial Velocity at Different Axial Sections (Turbulent case)

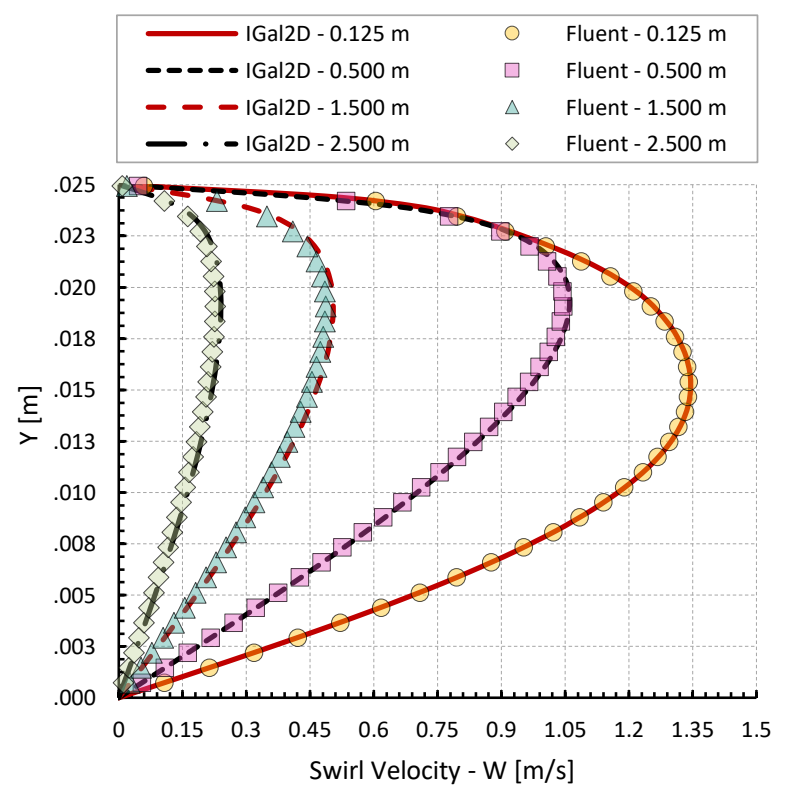
Figure 18 Distributions of Swirl Velocity at Different
Axial Sections (Turbulent case)

\section{CONCLUSIONS}

In this study, the axisymmetric IGal2D code of the Galatea-family software, is presented in brief and validated. In particular, it is evaluated against test cases considering inviscid and viscous, laminar or turbulent, swirling pipe flows. Attention is mainly directed toward the accurate prediction of velocity profiles in such cases, considering that the final goal of this work concerns corresponding simulations in shrouded wind turbines. Its results were compared with those of the commercial CFD software ANSYS Fluent. As an almost perfect agreement was succeeded against all the encountered test cases, the proposed methodology's potential to predict such complex flows in terms of accuracy is confirmed.

\section{ACKNOWLEDGMENTS}

This research has been financed by the Operational Program "Human Resources Development, Education and Lifelong Learning 2014-2020" and is co-financed by the European Union and Greek national funds (MIS: 5047362).

\section{REFERENCES}

Anderson, W.K., Rausch, R.D. and Bonhaus, D.L. (1996). Implicit/Multigrid Algorithms for Incompressible Turbulent Flows on Unstructured Grids. Computational Physics, 128, pp. 391-408, doi: 10.1006/jcph.1996.0219.

ANSYS Fluent 12.0, Theory Guide, 2009.

Blazek, J. (2001). Computational Fluid Dynamics: Principles and Applications. Elsevier Science, Kidlington, UK.

Koobus, B., Farhat, C. and Tran, H. (2000). Computation of Unsteady Viscous Flows around Moving Bodies Using the $k$ $\varepsilon$ Turbulence Model on Unstructured Dynamic Grids, Computer Methods in Applied Mechanics and Engineering, 190, pp. 1441-1466, doi: 10.1016/S0045-7825(00)00172-9.

Lee, H. and Lee, S. (2011). Convergence Characteristics of Upwind Method for Modified Artificial Compressibility Method. Aeronautical and Space Science, 12(4), pp. 318330, doi: 10.5139/IJASS. 2011.12.4.318.

Leloudas, S., Lygidakis, G., Eskantar, A. and Nikolos, I. (2020). A Robust Methodology for the Design Optimization of Diffuser Augmented Wind Turbine Shrouds. Renewable Energy, 150, pp.722-742, doi:10.1016/j.renene. 2019.12.098.

Leloudas, S., Eskantar, A., Lygidakis, G. and Nikolos, I. (2020). Low Reynolds Airfoil Family for Small Horizontal Axis Wind Turbines Based on RG15 Airfoil. SN Applied Sciences, 2:371, doi: 10.1007/s42452-020-2161-1.

Lygidakis, G., Sarakinos, S. and Nikolos I. (2016). Comparison of different agglomeration multigrid schemes for compressible and incompressible flow simulations. Advances in Engineering Software, 101, pp. 77-97, 137/071103-1, doi: 10.1016/j.advengsoft.2015.12.004.

Resiga, R., Muntean, S., Stein, P. and Avellan, F. (2009). Axisymmetric Swirling Flow Simulation of the Draft Tube Vortex in Francis Turbines at Partial Discharge. Fluid Machinery and Systems, 2(4), pp. 295-302, doi: 10.5293/IJFMS.2009.2.4.295.

Rocha, A.D., Bannwart, A.C. and Ganzarolli, M.M. (2015). Numerical and Experimental Study of an Axially Induced Swirling Pipe Flow. Heat and Fluid Flow, 53, pp. 81-90, doi: 10.1016/j.ijheatfluidflow.2015.02.003.

Sheng-Tao, Y. (1993). Convenient Method to Convert TwoDimensional CFD Codes into Axisymmetric Ones. Propulsion and Power, 9(3), pp. 493-495, doi: 10.2514/ 3.23649.

Singh, R.K., Ahmed, M.R., Zullah, M.A. and Lee, Y.H. (2012). Design of a Low Reynolds Number Airfoil for Small Horizontal Axis Wind Turbines. Renewable Energy, 42, pp. 66-76, doi: 10.1016/j.renene.2011.09.014.

Yang, H., Chen, J., Pang, X. and Chen, G. (2019). A New Aero-Structural Optimization Method for Wind Turbine Blades Used in Low Wind Speed Areas. Composite Structures, 207, pp. 446-459, doi: 10.1016/j.compstruct. 2018.09.050. 